\title{
La synonymie en question dans le cadre d'une sémantique constructiviste
}

Pierre Jalenques

\section{(2) OpenEdition}

1 Journals

\section{Édition électronique}

URL : https://journals.openedition.org/pratiques/1282

DOI : 10.4000/pratiques.1282

ISSN : 2425-2042

Éditeur

Centre de recherche sur les médiations (CREM)

\section{Édition imprimée}

Date de publication : 15 juin 2009

Pagination : 39-64

\section{Référence électronique}

Pierre Jalenques, "La synonymie en question dans le cadre d'une sémantique constructiviste », Pratiques [En ligne], 141-142 | 2009, mis en ligne le 20 juin 2014, consulté le 10 mars 2023. URL: http://journals.openedition.org/pratiques/1282 ; DOI : https://doi.org/10.4000/pratiques.1282 


\section{La synonymie en question dans le cadre d'une sémantique constructiviste}

\section{Pierre Jalenques}

Université de Rouen, LIDIFRA, EA 4305

Notre objectif dans cet article est de proposer une réflexion sur la synonymie dans le cadre d'une approche constructiviste du sens. Dans un premier temps, nous exposerons les grands principes de cette démarche du point de vue du sens des mots et des énoncés puis nous en tirerons les conséquences sur la façon dont on peut concevoir la synonymie. Nous défendrons l'idée que la synonymie est fondamentalement un phénomène relevant du niveau de l'énoncé. Dans un deuxième temps, nous proposerons une analyse constructiviste de la synonymie locale entre deux emplois de deux verbes du français, garder et laisser. Cela nous amènera à présenter au préalable une analyse sémantique de ces verbes.

\section{Introduction}

Nous partirons de la définition traditionnelle de la synonymie, admise à notre connaissance par la plupart des auteurs, à savoir que deux unités ${ }^{(1)}$ de la langue, $\mathrm{U} 1$ et $\mathrm{U} 2$, sont considérées comme synonymes si et seulement si ( $c f$. par exemple Auroux, 1984) ${ }^{(2)}$ :

(1) J'emploie le terme unité pour désigner tout élément de la langue, qu'il s'agisse d'un mot ou bien d'un morphème, puisque, par exemple, les affixes dérivationnels sont concernés par le phénomène de la synonymie.

(2) Cette définition traditionnelle est souvent assortie d'un troisième critère, à savoir que U1 et U2 doivent appartenir à la même catégorie ( $c f$. M. Gross (1997 : 72). Pour cette raison, des mots comme progresser et progression ne sont pas considérés comme synonymes. Cependant, en pratique, en particulier dans le domaine grammatical, ce troisième critère n'est souvent pas respecté. Ainsi, la conjonction de coordination car est généralement donnée par les dictionnaires comme synonyme de la conjonction de subordination parce que ; 1'adverbe ensuite est donné comme synonyme de la préposition après; les adverbes de nouveau ou encore sont souvent considérés comme synonymes du préfixe RE ( $c f$. Fuchs, 1992), etc. Pour une hypothèse défendant la non synonymie entre des mots comme progresser / progression, voir Sanchez et Cadiot (2007 : 115). 
a) U1 et U2 ont un sens équivalent, à quelques nuances près ${ }^{(3)}$;

b) ces unités peuvent se substituer l'une à l'autre, c'est-à-dire :

i) apparaître dans le même co-texte (généralement un énoncé) ${ }^{(4)}$

ii) la commutation de U1 avec U2 ne change pas le sens de l'énoncé, à quelques nuances près

Cette définition n'est pas neutre sur le plan théorique. Elle engage, généralement de façon implicite, plusieurs postulats en sémantique. Observons tout d'abord que la conjonction de ces deux critères semble traduire une hésitation, voire une contradiction, sur le statut même de la synonymie. Le critère a) laisse supposer que la synonymie est conçue comme une relation entre deux mots considérés hors emploi, donc comme un phénomène de langue (comme un phénomène relevant du système de la langue, dans une vision structuraliste). Au contraire, le critère b) laisse supposer que la synonymie ne concerne que les mots en emploi, puisque le test de substitution consiste à envisager les deux unités dans un co-texte donné ; il s'agirait dans ce cas d'un phénomène de discours.

Or, on le sait, la plupart des unités de la langue (en tout cas celles de la langue courante) donnent lieu à des emplois sémantiquement très divers (ce que 1'on appelle traditionnellement la polysémie). Donc, de façon générale, la relation de synonymie concerne des unités dites polysémiques. Dès lors, si on envisage la synonymie comme une relation entre des mots considérés hors emploi (ce que défendent de fait la plupart des dictionnaires de synonymes actuels), cela revient à admettre que les différentes significations intuitivement associées à une unité sont elles-mêmes concevables hors emploi, ce qui est sujet à débat en sémantique. Toute prise de position sur la synonymie de ce point de vue impose donc, implicitement ou explicitement, une prise de position sur le phénomène général de la polysémie.

De plus, toute option théorique sur la polysémie conduit, implicitement ou explicitement, à une prise de position sur la compositionnalité du sens des énoncés, c'est-à-dire sur le rapport entre le sens global d'un énoncé et le sens des mots et morphèmes qui le constituent. En effet, défendre l'idée que les diverses significations d'une unité peuvent être considérées hors emploi revient à admettre que ces significations sont constituées indépendamment des co-textes où elles se manifestent. Autrement dit, le sens d'un mot tel qu'il est perçu dans un énoncé constitue une partie de sens indépendante et disjointe du sens global de cet énoncé. Là encore, il s'agit d'une affirmation sujette à débat en sémantique.

Ainsi, nous considérons que les différentes significations intuitivement associées à une unité impliquent nécessairement la prise en compte de ses co-textes d'emplois (éventuellement implicites) et que ces significations ne sont pas indépendantes du sens global des énoncés dans lesquels 1'unité apparaît. En conséquence, nous défendrons l'idée que la synonymie est un phénomène de discours.

Nous illustrerons notre propos à partir des emplois des verbes garder et laisser.

(3) Je ne rediscuterai pas ici du fait, admis par tous les auteurs, que l'identité de sens totale, absolue entre deux unités ne se rencontre guère dans les langues et donc lorsque nous parlons de synonymie nous parlons toujours d'équivalence sémantique relative.

(4) Par convention, j'appellerai co-texte l'environnement textuel d'une unité ; il s'agira généralement d'un énoncé, mais selon les cas, le co-texte pourra correspondre à une suite de mots plus large ou plus restreinte qu'un énoncé. J'appellerai contexte la situation à laquelle renvoie un énoncé. 
Nous avons retenu ces deux mots d'une part pour leur polysémie importante mais aussi car l'existence d'une synonymie entre ces deux verbes est a priori contre intuitive. Considérés hors emploi, ces verbes sont plutôt perçus comme antonymes. Leur synonymie ne va donc pas de soi a priori. Un de nos objectifs étant de montrer que les relations sémantiques preçues entre les mots ne sont pas données (hors emploi) mais son construites (en emploi), ces deux verbes nous ont paru être de bons candidats pour montrer que la relation de synonymie est une relation construite en co-texte. Voici donc deux types d'emplois où ces deux verbes peuvent être perçus comme synonymes :

(1) a. On préfère garder les volets fermés à cause de la chaleur

b. On préfère laisser les volets fermés à cause de la chaleur

(2) a. on est sympa, on t'a gardé une part de gâteau !

b. on est sympa, on t'a laissé une part de gâteau !

Du point de vue de l'antonymie, nous avons par exemple :

(3) a. pendant la visite de la base, vous devez garder vos papiers d'identité

b. pendant la visite de la base, vous devez laisser vos papiers d'identité

Ces deux verbes ont par ailleurs des emplois dont les interprétations sont relativement éloignées. L'emploi de l'un ou l'autre verbe donne lieu à une séquence difficilement interprétable :

(4) a. hier soir, Marie a gardé les enfants de la voisine

b. ? hier soir, Marie a laissé les enfants de la voisine

En (4a), l'interprétation est proche de «s'occuper de et surveiller les enfants »; en $(4 b)$ le problème n'est pas tant que la séquence serait agrammaticale; elle est surtout peu interprétable. Elle devient interprétable si on ajoute une infinitive :

(4') b. hier soir, Marie a laissé les enfants de la voisine regarder la télé jusqu'à $22 \mathrm{~h}$

Mais l'interprétation qui émerge n'est ni synonyme ni antonyme de celle que l'on a en (4a): «permettre / autoriser une situation pour les enfants » est simplement une valeur sémantique différente de «s'occuper de et surveiller les enfants».

\section{La synonymie dans le cadre d'une sémantique constructiviste}

\subsection{Principes généraux d'une approche constructiviste}

Le point de vue que nous défendons ici se rattache au cadre de la théorie des opérations prédicatives et énonciatives (désormais T.O.P.E) développée par Antoine Culioli ${ }^{(5)}$. L'objectif ici n'est pas d'aborder les concepts de cette théorie mais de présenter les principes d'analyses et la conception du sens des énoncés qui lui sont attachés. Nous illustrerons notre approche à partir du verbe garder. Le dictionnaire Petit Robert (2007) recense dix-sept emplois pour ce verbe. Considérons les trois emplois suivants :

(5) Cf. Culioli (1990-1999). L'approche défendue ici est constructiviste sur plusieurs plans : d'une part du point de vue de la relation entre langage et cognition, d'autre part du point de vue du sens des mots et des énoncés. Nous n'aborderons ici que le second aspect. Pour une présentation plus complète de ce que l'on entend par constructivisme dans la T.O.P.E., je renvoie à Franckel (2002). 
(5) a. Pendant la cérémonie, Paul a gardé son manteau

b. On préfère garder les volets fermés à cause de la chaleur

c. Hier soir, Paul a gardé les enfants de sa sœur

En (5a) on associe intuitivement à garder un sens proche de « continuer à avoir sur soi, continuer à porter, ne pas enlever, etc. »; en (5b), on associe intuitivement à garder un sens proche de « maintenir qqch. dans un certain état; enfin en (5c), on associe intuitivement à garder un sens proche de « surveiller un enfant pour veiller à ce qu'il ne lui arrive rien de fâcheux ». Convenons d'appeler acceptions ces différentes significations intuitivement associées à la présence de garder dans l'énoncé.

Dans notre approche constructiviste, chacune de ces acceptions résulte non pas de la présence de garder mais résulte des interactions entre le verbe garder et les autres mots et morphèmes de l'énoncé (qui, généralement, sont eux-mêmes polysémiques). En d'autres termes, dans la séquence (5a), la formulation «continuer à avoir sur soi » ne constitue pas une description sémantique du verbe garder lui-même mais constitue une description de ce qui résulte de l'interaction entre garder et le reste de l'énoncé.

Sur ce point, la T.O.P.E. est proche du point de vue holiste qui considère que le sens d'un mot dans un énoncé est au moins en partie déterminé par le sens global de l'énoncé ; cela veut dire que le contenu de l'acception elle-même est en partie constitué à partir des propriétés sémantiques des mots environnant le mot considéré. Le point de vue inverse correspond à l'approche généralement appelée atomiste, qui considère que si le sens du tout résulte de la composition du sens des parties, par contre le sens des parties ne dépend pas du sens du tout; bref, les acceptions sont constituées indépendamment du co-texte, donc hors co-texte ${ }^{(6)}$.

Revenons à l'approche constructiviste. Reprenons l'acception en (5a) que l'on peut scinder pour les besoins de l'exposé en deux parties :

- continuer à avoir

- sur soi

On pourrait considérer que la propriété « sur soi » découle du sens du complément du verbe (manteau). Quand dans une situation donnée, on continue à avoir un vêtement, on comprend a priori qu'on a le vêtement sur soi, car un vêtement se porte. Bien sûr dans une analyse sémantique globale de l'énoncé, il faudrait aussi rendre compte pour manteau de l'émergence de l'interprétation vêtement plutôt que celle que l'on a dans un manteau neigeux ou dans le manteau terrestre; idem pour les autres unités de l'énoncé.

Ceci dit, nous constatons que si l'on remplace manteau par vieux journaux, par exemple dans pourquoi tu gardes tous ces vieux journaux, la propriété «sur soi » disparaît. Dans ce cas, on pourrait conclure qu'en fait le sens de garder correspond à une sous-partie de l'acception qu'on lui associe intuitivement, à savoir « continuer à avoir ». Cette analyse ne marche pas comme le montre la comparaison avec l'exemple (5') :

(5') ? Pendant la cérémonie, Paul a tenu son manteau

Certes cette séquence n'est pas très naturelle. Elle passerait mieux si on envisa-

(6) Pour une discussion argumentée entre les points de vue atomiste et holiste, je renvoie à Gosselin (1996). 
geait que la cérémonie a lieu en plein air, qu'il y a un vent très fort, si on ajoutait l'adverbe fermement et s'il s'agissait d'un chapeau plutôt que d'un manteau. Peu importe. Ce qui compte ici, c'est d'observer qu'avec tenir, l'idée que Paul porte son manteau n'est plus du tout saillante; il peut l'avoir à la main, il peut l'avoir posé sur une chaise à côté de lui et avoir une main posée dessus, etc. ${ }^{(7)}$ Ce contraste fait apparaître que garder y est pour quelque chose dans l'émergence de la propriété « sur soi » dans l'énoncé (5a); mais en même temps cette propriété ne lui est pas intrinsèque puisqu'on ne la retrouve pas dans d'autres co-textes. Le complément pendant la cérémonie n'est pas neutre vis-à-vis de l'interprétation de la relation entre garder et manteau. Avec un autre complément circonstanciel, la propriété « sur soi » disparaît : Paul garde son manteau dans une armoire fermée à double tour (imaginons qu'il s'agisse d'un manteau précieux auquel il tient beaucoup). Le verbe garder participe à la construction de l'interprétation «sur soi » avec certains co-textes mais il n'est pas porteur de cette valeur.

C'est en cela que l'approche que nous défendons est constructiviste ${ }^{(8)}$ : la propriété « sur soi » ne correspond pas à un simple ajout sémantique apporté par le cotexte ; et garder n'est pas constitué par un noyau de sens sous-déterminé qui recevrait de son co-texte des propriétés sémantiques supplémentaires. La propriété «sur soi » résulte / émerge de l'interaction entre garder et les autres éléments de l'énoncé, dont manteau. De la même façon, on pourrait montrer que l'autre partie de l'acception « continuer à avoir » est elle-même construite (nous y reviendrons dans la deuxième partie de l'article). En conséquence, l'acception que nous percevons en tant que partie du sens de l'énoncé associée à garder est elle-même globalement construite, tout autant que le sens de l'énoncé lui-même est construit.

Enfin, c'est une chose de percevoir intuitivement des parties de sens dans la signification globale d'un énoncé ; cela en est une autre de considérer que telle partie du sens global de l'énoncé correspond directement au sens de tel mot ou tel morphème. Considérons la séquence suivante, par exemple à propos d'un couple de retraités qui possédait plusieurs restaurants; ils ne les ont pas tous vendus :

(6) Ils ont gardé un petit resto rue des Ecoles

Sur le plan aspectuo-temporel, on comprend que les effets du procès durent dans le présent (le couple est toujours à l'heure actuelle propriétaire du restaurant). On pourrait être tenté d'attribuer cette partie du sens de l'énoncé au passé composé. Mais considérons maintenant la séquence suivante :

(6') Ils ont tenu un petit resto rue des Ecoles

(7) On retrouve le même type de différence dans le contraste entre les deux séquences suivantes :

a. Pour le passage du gué, je vous conseille de garder vos chaussures

b. Pour le passage du gué, je vous conseille de (bien) tenir vos chaussures.

En a., on a les chaussures « sur soi » au sens où on les a aux pieds, alors qu'en b. on les a $a$ priori à la main.

(8) Notre approche est également constructiviste au sens où, à co-texte constant, on considère qu'un mot peut avoir différents types d'interactions avec ce co-texte, avec pour résultat la construction de différentes valeurs interprétatives. De Voguë et Paillard (1997) analysent ainsi les différentes interprétations possibles d'une séquence comme Pierre suit Paul, où il peut s'agir par exemple d'une filature ou bien d'une relation de succession entre deux noms dans une liste. 
Le sens du procès en jeu change, mais également la valeur aspectuo-temporelle. Ici, les effets du procès ne se prolongent pas dans le présent. Comme la seule différence entre (6) et (6') concerne le verbe lui-même, on pourrait alors considérer que c'est lui qui est porteur de la dimension aspectuo-temporelle de l'énoncé. Mais cette hypothèse ne tient pas non plus puisque si je remplace le passé composé par l'imparfait je vais obtenir d'autres valeurs aspectuo-temporelles. Je suis obligé de conclure que la partie du sens global de l'énoncé constituée par sa dimension aspectuo-temporelle résulte de l'interaction entre différents éléments de l'énoncé, dont le verbe. Autre exemple, repris à Culioli : lorsque on emploie le verbe aimer au conditionnel présent, une valeur proche de vouloir émerge (j'aimerais bien un café / ? j'aime bien un café). Il n'y a donc pas de correspondance directe entre les parties du sens de l'énoncé et les parties de l'énoncé (les mots, les morphèmes, les constructions syntaxiques qui le constituent). C'est à ce propos que J.-J. Franckel ( $c f$. par exemple Franckel, 2005) parle de contamination contextuelle, désignant par là l'illusion de perception consistant à attribuer à une unité une valeur supposée liée à sa présence alors que cette valeur est construite par l'ensemble de l'énoncé. Bref, l'illusion réside dans l'attribution d'une propriété du tout à une partie de ce tout.

Plus précisément, dans la T.O.P.E., le sens d'un énoncé est pensé comme le résultat d'un enchaînement d'opérations langagières. Dès lors, les parties de sens que l'on perçoit intuitivement correspondent à des parties du résultat interprétatif et non à des parties des opérations ayant conduit à ce résultat.

C'est en ce sens que nous avons dit ci-avant que la formulation de l'acception pour (5a) constitue une description de ce qui résulte de l'interaction entre garder et le reste de l'énoncé. Dans cette optique, une description lexicographique qui recense et décrit les différentes acceptions possibles pour garder ne constitue pas une description du verbe garder lui-même, mais constitue une description des propriétés sémantiques émergeant des interactions du verbe garder avec divers énoncés types (éventuellement implicites) ${ }^{(9)}$.

Ainsi, l'identité du verbe garder correspond à un en-deçà de toutes les acceptions qu'on peut intuitivement lui associer. Par hypothèse, cet en-deçà est invariant; on le retrouve donc dans tous les emplois du verbe. Dans la théorie des opérations prédicatives et énonciatives, cet invariant est appelé forme schématique (notée F.S.). "C'est un schéma parce qu'il organise le co-texte, et une forme parce qu'il en tire sa substance » (de Voguë, Paillard, $1997: 43$ ). Cette F.S. « représente l'identité de l'unité. Elle constitue le cadre d'un raisonnement permettant de dégager le rôle respectif de l'unité et de son co-texte dans la variation des sens qui peuvent lui être associés » (Franckel : $2002: 12$ ).

Cette F.S. invariante n'est donc pas un noyau de sens abstrait sous-déterminé qui recevrait des propriétés supplémentaires de la part de son co-texte; ce n'est pas un « chapeau sémantique » pour reprendre une formulation critique récurrente de G. Kleiber à l'encontre des approches proposant des invariants sémantiques ( $c f$. Kleiber 2008 : 92). Cet invariant est constitué par la façon dont garder participe à la construction du sens des énoncés où il apparaît ${ }^{(10)}$. Cette idée se précisera en seconde partie lorsque nous présenterons une hypothèse d'invariant pour garder.

(9) Dans un cadre différent, M. Gross (1997: 74) défend un point de vue similaire : « un mot est mentalement lié à des contextes non explicites ».

(10) Pour une présentation plus précise du concept de forme schématique, je renvoie à de Voguë et Paillard (1997). 


\subsection{Conséquences pour l'analyse théorique de la synonymie}

Considérons maintenant trois emplois du verbe laisser (le dictionnaire Petit Robert (2007) en recense treize).

(7) a. les manifestants ont laissé passer la délégation

b. François a laissé les clés au gardien

c. On préfère laisser les volets fermés à cause de la chaleur

En (7a) l'acception intuitivement associée à laisser correspond a peu près à « ne pas empêcher, ne pas faire obstacle à un événement »; en (7b), on associe intuitivement à laisser un sens proche de « confier ; enfin en $(7 \mathrm{c})$, on associe intuitivement au verbe un sens proche de «maintenir qqch dans un certain état ». Reprenons la séquence $(5 \mathrm{~b})$ :

(5) b. On préfère garder les volets fermés à cause de la chaleur

Nous constatons qu'en (5b), garder est perçu comme sémantiquement similaire, donc synonyme, du verbe laisser avec lequel il est en relation de substitution (puisqu'ils peuvent apparaître dans le même co-texte sans changement de sens majeur pour l'énoncé). Mais, lorsqu'on affirme que dans le co-texte (5b)/(7c), il y a une synonymie entre garder et laisser, de quoi parle-t-on exactement ?

La relation de synonymie intuitivement perçue concerne une des acceptions auxquelles leurs emplois donnent lieu. Or, dans la perspective constructiviste que nous défendons, deux plans sont distingués : celui des formes schématiques (F.S.) et celui des acceptions construites en co-texte. Convenons de désigner par $\mathrm{a}_{1} \mathrm{a}_{2} \mathrm{a}_{3}$ les trois acceptions correspondant aux emplois (5a-c) de garder et convenons de désigner par a' $1{ }_{1}{ }^{\prime}{ }_{2} a^{\prime}{ }_{3}$ les trois acceptions correspondant aux emplois (7a-c) de laisser. Nous avons affaire à la situation suivante ${ }^{(11)}$ :

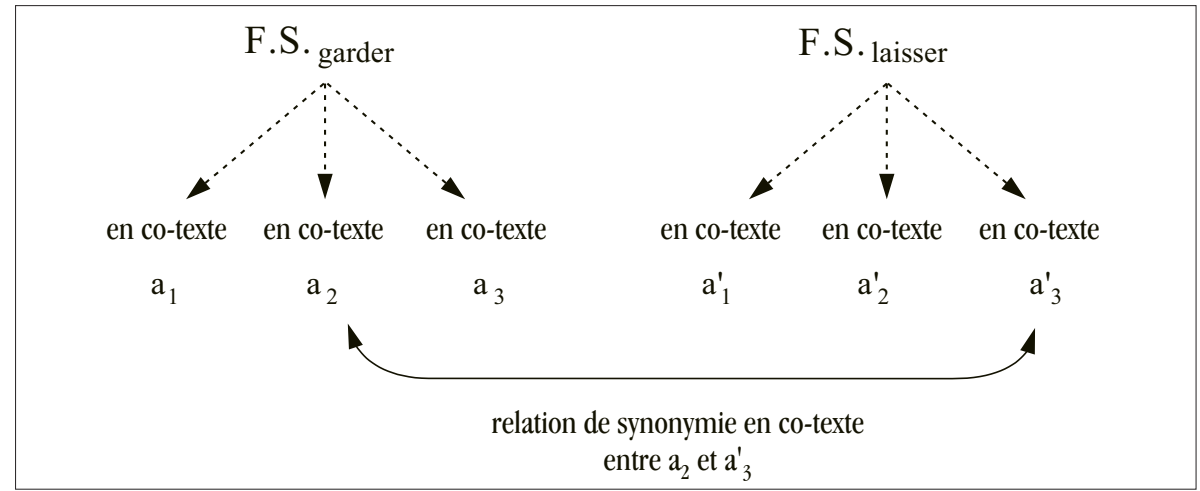

(11) En représentant le rapport de la F.S. aux acceptions par des lignes en pointillés, je voudrais éviter à nouveau un éventuel malentendu : le « chemin » qui conduit d'une F.S. à une acception est un chemin de construction du sens; ce n'est donc pas un chemin direct dans la mesure où de nombreux facteurs peuvent intervenir simultanément dans la construction d'une acception, d'une part les F.S. des autres mots de l'énoncé mais aussi la valence du verbe, la nature syntaxique des compléments, la détermination des compléments, les marqueurs aspectuotemporels, etc. Comparer par exemple l'acception intuitivement associée au verbe passer dans passer la question 3 et passer à la question 3 ou bien les acceptions intuitivement associées au verbe savoir selon la nature syntaxique de son complément: je sais que je cours vite ( j'en ai conscience / j'en suis convaincu); je sais courir vite ( $\sim \mathrm{j}$ 'en suis capable). 
Nous avons défendu l'idée que les acceptions ne constituent pas des propriétés du mot mais des propriétés émergentes en co-texte. La relation de synonymie ne concerne donc pas les mots eux-mêmes, c'est-à-dire leurs formes schématiques. Ceci a deux conséquences:

- la synonymie est un phénomène de discours : la relation de synonymie implique la présence d'un co-texte d'emploi ${ }^{(12)}$;

- dans la mesure où le co-texte est partie prenante dans l'émergence de l'acception considérée, il est donc impliqué dans la relation de synonymie entre les deux acceptions; en conséquence la relation de synonymie concerne non pas le seul mot garder / laisser mais concerne l'ensemble constitué par le mot et son co-texte, c'est-à-dire l'énoncé dans son ensemble.

Les exemples (5b) et (7c) n'illustrent pas une proximité sémantique entre garder et laisser eux-mêmes mais illustrent une proximité entre un aspect de l'interprétation globale d'un énoncé et un aspect de l'interprétation globale d'un autre énoncé qui se distinguent formellement par la commutation de garder et laisser. Or, considérer une proximité sémantique entre deux énoncés nous renvoie au phénomène bien connu de la paraphrase ${ }^{(13)}$. Je soutiens l'idée qu'il n'y a pas deux ordres de phénomènes, la synonymie de mots et la synonymie de phrases, mais un seul phénomène, la paraphrase. Ce que l'on entend traditionnellement par synonymie de mots correspond à un point de vue particulier sur l'analyse de paraphrases, en l'occurrence un point de vue où l'on s'intéresse à une partie du sens construit de ces paraphrases, mais le phénomène en jeu concerne bien les paraphrases elles-mêmes.

Dans notre approche constructiviste, si la synonymie de mots devait exister, elle concernerait la relation entre les formes schématiques elles-mêmes, et non les acceptions construites en co-texte à partir de ces formes schématiques. Or, c'est un fait bien connu, deux unités dites polysémiques ne donnent lieu à synonymie que pour une partie des acceptions qu'on leur associe intuitivement ; bien souvent, la synonymie ne concerne même qu'une seule des acceptions ${ }^{(14)}$. Sur les dix-sept acceptions associées à garder, seules deux d'entre elles donnent lieu à une synonymie avec une acception de laisser. Outre celle vue ci-avant, il y a l'acception proche de « réserver qqch. pour qqn » que nous avions vu en introduction ${ }^{(15)}$ :

(2) a. on est sympa, on t'a gardé une part de gâteau

b. on est sympa, on t'a laissé une part de gâteau

Si garder et laisser ne donnent lieu à des énoncés synonymes que pour deux de leurs emplois (sur dix-sept pour garder, sur treize pour laisser), cela implique que pour l'essentiel ces verbes sont sémantiquement différents. Dès lors, on peut supposer que leurs formes schématiques ne sont pas synonymes. C'est ce que nous verrons dans la deuxième partie de l'article.

(12) Sur ce point, nous rejoignons Sanchez et Cadiot (2007).

(13) $C f$. par exemple, C. Fuchs (1994: 46) : «on peut concevoir la relation sémantique entre paraphrases comme une relation de synonymie ", où synonymie est entendue au sens d'une relation d'équivalence sémantique partielle. Voir également G. Gross (1997: 73).

(14) $C f$. par exemple Duchàcek (1964); François, Victorri et Manguin, (2003); Fuchs (2007).

(15) On observe également une proximité interprétative pour une variante pronominale de cette classe d'emplois :

- je propose que, cet après-midi, on se laisse un peu de temps pour traiter le problème

- je propose que, cet après-midi, on se garde un peu de temps pour traiter le problème 
Comme la situation de garder et laisser correspond au cas général (pour la plupart des couples d'unités, la synonymie ne concerne qu'une petite partie de leurs acceptions), on pourrait d'emblée conclure que la synonymie n'existe pas sur le plan des formes schématiques (les invariants associés aux unités dans notre théorie) et donc que, dans notre approche constructiviste, la synonymie entre des mots (ou morphème) n'existe pas. Mais, le degré de différence entre des acceptions n'étant pas proportionnel au degré de différence entre les deux formes schématiques qui participent à leur construction, on peut, dans le principe, parfaitement envisager des formes schématiques équivalentes dans la plupart de leurs aspects et dont la différence a priori ténue pourrait donner lieu à la construction en co-texte d'acceptions perçues comme très différentes ${ }^{(16)}$. La possibilité d'équivalences importantes entre formes schématiques reste me semble-t-il une question ouverte. Tout ce que je peux affirmer, c'est que je n'en connais aucun exemple (mais le nombre de verbes du français pour lesquels une forme schématique a été proposée ne dépasse pas quelques dizaines).

Remarquons que l'approche atomiste rencontre ici un problème pour lequel ses défenseurs ne proposent pas de solution, à ma connaissance. Imaginons pour fixer les idées un cas de figure où les différents emplois de deux unités U1 et U2 donnent lieu à dix acceptions pour chacune et ne donnent lieu à synonymie que pour une de ces dix acceptions. En conséquence, pour neuf acceptions sur dix, ces deux unités ne sont pas synonymes. On peut donc affirmer que, pour l'essentiel, elles sont sémantiquement différentes. Mais, comme en même temps elles sont synonymes, disons pour leurs acceptions $\mathrm{a}_{1}$ et $\mathrm{a}{ }_{1}$, et que la synonymie correspond dans cette approche à la situation où les mots eux-mêmes sont pour l'essentiel sémantiquement équivalents à quelques nuances près, on est obligé de conclure que les unités U1 et U2 sont à la fois pour l'essentiel sémantiquement équivalentes (en vertu de leur synonymie) et pour l'essentiel sémantiquement différentes (en vertu de leur polysémie). Il y a là une contradiction me semble-t-il difficile à résoudre dans le cadre d'une approche atomiste.

Toujours dans le cadre atomiste, le fait même que la relation de synonymie entre deux unités ne concerne qu'une petite partie de leurs acceptions est en soi étonnant. Considérons les acceptions $\mathrm{a}_{1}$ et a' ${ }_{1}$ perçues comme synonymes de l'exemple ci avant. Elles sont par hypothèse sémantiquement équivalentes à quelques nuances près. Ces nuances constituent donc la différence sémantique entre $\mathrm{a}_{1}$ et $\mathrm{a}^{\prime}{ }_{1}$. Notons D cette différence sémantique. Comment se fait-il que cette même différence $\mathrm{D}$ ne se retrouve pas entre $\mathrm{a}_{2}$ et $\mathrm{a}_{2}{ }_{2}$, entre $\mathrm{a}_{3}$ et a' ${ }_{3}$, etc. ? C'est-à-dire comment se fait-il que le différentiel sémantique D entre U1 et U2 ne soit pas le même pour toutes leurs acceptions? Et comment se fait-il que l'on ne rencontre guère de telles unités dans les langues?

Au contraire, dans notre approche constructiviste, la situation s'explique naturellement. La synonymie entre les acceptions $\mathrm{a}_{1}$ et $\mathrm{a}_{1}{ }_{1}$ concerne un énoncé contenant U1 ou U2 et non les unités U1 et U2 elles-mêmes. Dès lors, ce sont les acceptions construites en co-texte qui sont sémantiquement équivalentes à quelques nuances près et non les formes schématiques de U1 et U2. Ces F.S. sont a priori très différentes; d'où le fait qu'en dehors du co-texte considéré, les emplois des unités U1 et U2 donnent lieu à des acceptions sémantiquement très différentes.

(16) Dans la même optique, Franckel et Lebaud (1990: 12) ont montré que « le fonctionnement d'un verbe [c'est-à-dire sa F.S.] comme désirer est à certains égards beaucoup plus proche de celui de entendre qu'il ne l'est de vouloir ou de souhaiter auxquels il peut pourtant se substituer sans changement de sens apparent dans certains énoncés ». 


\subsection{Synonymie et substitution}

Nous avons rappelé en introduction que le test de substitution constitue le critère principal pour définir traditionnellement la synonymie, en dehors de l'intuition directe d'une proximité sémantique entre deux unités. Nous voudrions montrer ici que l'usage même de ce critère pour définir la synonymie repose sur un postulat atomiste. Rappelons que deux unités U1 et U2 sont en relation de substitution, si et seulement si :

i) elles apparaissent dans le même co-texte (généralement un énoncé)

ii) la commutation de U1 avec U2 ne change pas le sens de l'énoncé, à quelques nuances près

Considérons deux énoncés E1 et E2 qui ne se distinguent formellement que par la commutation des unités U1 et U2. Le premier postulat implicite consiste à admettre que 1'équivalence sémantique entre E1 et E2 implique l'équivalence sémantique entre U1 et U2. C'est le fondement même du test de substitution. Cela revient à affirmer qu'il y a une correspondance directe entre le sens du tout et le sens de la partie.

Dans une approche non atomiste, on peut parfaitement envisager que des parties différentes contribuent à un même résultat interprétatif global. C'est précisément ce que nous montrerons avec garder / laisser en deuxième partie. Prenons deux exemples à travers un bref détour par 1'arithmétique. Considérons la séquence $(X)^{2}+3$, où $X$ représente une variable. Faisons commuter les nombres +2 et -2 à la place de $X:(+2)^{2}+3=(-2)^{2}+3=7$. Bref, que l'on remplace la variable $X$ par +2 ou bien par -2 , le résultat est le même (car « moins par moins égal plus »). Deux nombres opposés, dans une même formule, peuvent conduire au même résultat. Prenons un autre exemple, où cette fois nous faisons commuter des opérations. Dans le co-texte 2_2, les opérations d'addition et de multiplication conduisent au même résultat : $2+2=2 * 2=4$. Intuitivement, elles apparaissent donc comme localement « synonymes ». Le point essentiel est que la relation d'identité concerne le résultat auquel conduisent les opérations dans ce co-texte et non les opérations elles-mêmes. De même, la synonymie intuitivement perçue entre garder et laisser concerne le résultat interprétatif obtenu à travers leur relation au co-texte et non leurs formes schématiques ayant participé à la construction de ce résultat interprétatif. En conséquence, dans notre perspective constructiviste, l'équivalence sémantique des énoncés E1 et E2 n'implique en aucun cas l'équivalence des unités U1 et U2. Le recours au test de substitution comme «preuve » d'une synonymie entre les mots eux-mêmes ne tient pas.

Le second postulat implicite repose sur la corrélation supposée entre identité distributionnelle et identité sémantique. En effet, on admet généralement que deux unités totalement synonymes seraient substituables l'une à l'autre dans tous leurs co-textes, ce qui revient à admettre qu'elles auraient exactement les même co-textes, c'est-à-dire la même distribution. Dans le même temps, on admet que des unités qui ne seraient sémantiquement équivalentes qu'à quelques nuances près n'auraient que partiellement la même distribution. Cela revient à postuler que plus deux unités sont sémantiquement proches et plus elles seraient distributionnellement proches. Le postulat est donc le suivant : le degré de proximité distributionnelle entre deux unités est proportionnel à leur degré de proximité séman- 
tique. Ce postulat est issu de 1'analyse distributionnelle ${ }^{(17)}$. Il relève également d'une approche atomiste.

En effet, la proximité sémantique dont il est question ici, comme précédemment, concerne les acceptions construites en co-texte et non les formes schématiques associées aux mots eux-mêmes. Or, le degré de proximité sémantique entre les acceptions $\mathrm{a}_{1}$ et a' ${ }_{1}$ découle également de la présence des éléments du co-texte autour de U1 ou U2. Par contre, les propriétés distributionnelles dont il est question concernent bien les unités U1 et U2 elles-mêmes. Le postulat ci-dessus revient à donc à établir une corrélation entre la distribution des unités U1 / U2 et une ou plusieurs de leurs acceptions alors même que ces acceptions sont construites également à partir d'autres éléments que U1 / U2. Si l'on peut montrer qu'une telle corrélation n'existe guère, cela constituera un argument en faveur de l'approche constructiviste.

Observons tout d'abord que, y compris dans leurs emplois perçus comme synonymes, bien des mots, en particulier des verbes, n'apparaissent pas exactement dans le même co-texte ${ }^{(18)}$. Ainsi, le verbe ressembler qui se construit avec la préposition à a pour synonyme tenir de, ou bien imiter qui se construit sans préposition. Le verbe arriver, transitif indirect est donné comme synonyme de atteindre, transitif direct. Enfin, considérons le verbe reprocher, qui rentre dans la construction verbe $S N$ à $S N$. Sur les vingt-trois synonymes de ce verbe proposés dans le Dictionnaire Electronique des Synonymes du laboratoire Crisco, seuls imputer et remontrer entrent dans la même construction syntaxique que reprocher. Autre exemple avec un adjectif : grand dans une grande attention est donné comme synonyme de soutenu alors que ce dernier, contrairement à grand apparaît après le nom : une attention soutenue /* une soutenue attention. En toute rigueur, tous ces synonymes ne se substituent donc pas les uns aux autres, à moins d'affaiblir le test de substitution en exigeant que les synonymes apparaissent «à peu près » dans le même co-texte ; mais ce faisant, on vide ce test de sa substance (atomiste).

Ajoutons qu'en morphologie dérivationnelle où la synonymie constitue un phénomène massif, dans la plupart des cas, les affixes synonymes ne se combinent pas avec les mêmes bases. Les préfixes suivants évoquent tous l'idée de négation :

- in : incorrect, inadmissible, etc.

- a : anormal, apolitique, agrammatical, asymétrique, etc.

- dé : déloyal, défavorable, désagréable, désintéressé, etc.

- mal : malhabile, malcommode, malodorant, etc.

Nous n'avons pas dénormal, dégrammatical ni acorrect ou infavorable. Ainsi, en morphologie dérivationnelle, la relation de synonymie se manifeste généralement à co-textes différents ${ }^{(19)}$.

(17) «If we consider words or morphems A and B to be more different in meaning than A and C, then we will often find that the distributions of A and B are more different than the distribution of $\mathrm{A}$ and $\mathrm{C}$. In other words, difference of meaning correlates with difference of distribution » (Harris, $1954: 156)$.

(18) Nous rejoignons sur ce point les observations de Lipshitz (1978). De même, R. Schreyer (1976 : 3) met en doute la validité de ce test tout en lui reconnaissant une utilité pratique, selon nous illusoire : «the assumption that it is easier for an informant to decide on the sameness of sentence meaning than on that of shorter expressions ").

(19) Bien sûr, la notion de négation est complexe et hétérogène et la proximité sémantique à laquelle donnent lieu les emplois de ces préfixes est relative. C'est précisément parce qu'ils ne marquent pas le même type de négation que ces préfixes se combinent à des bases différentes. 
Comparons maintenant plus en détail la distribution de quelques verbes. Considérons un exemple bien connu de synonymes, les verbes savoir et connaître qui posent souvent des difficultés aux apprenants étrangers. Leur emploi peut donner lieu à des énoncés sémantiquement proches :

(8) a. est-ce que tu sais ta leçon?

b. est-ce que tu connais ta leçon?

Examinons maintenant les distributions respectives de ces deux verbes; d'abord sur le plan syntaxique. On le sait, le verbe savoir prend essentiellement comme complément des propositions subordonnées et plus rarement des SN, alors que le verbe connaître n'admet jamais de complément phrastique et prend comme complément essentiellement des $\mathrm{SN}^{(20)}$ :

(9) a. je sais / * connais que Marie t'a appelé

b. je ne sais $/ *$ connais pas si je viendrai

c. je ne sais $/ *$ connais pas qui est venu

d. je sais $/ *$ connais me montrer très généreux

e. je sais / connais ton amitié pour Marie

Donc, sur le plan syntaxique, leur distribution ne se recoupe que lorsque le complément est un SN. Examinons maintenant les contraintes interprétatives sur le complément. Le verbe connaître admet comme complément des SN renvoyant à des types d'entités très variables, comme des animés humains (je connais Paul), des lieux (je connais cet endroit), des notions abstraites (je connais son penchant pour l'alcool), des œuvres artistiques (tu connais ce tableau / ce film / ce livre?), des situations (il a connu la faim / l'humiliation / des revers), etc. Le verbe savoir n'admet comme complément quasiment aucun de ces types d'entités à part quelques notions abstraites. Enfin, le verbe connaître admet également comme premier argument des entités autre que animé humain (notre projet a connu un vif suc$c e ̀ s)$, contrairement à savoir. En résumé, si l'on combine les paramètres syntaxiques et lexicaux, il apparaît que la distribution de ces deux verbes est totalement différente, à quelques exceptions près.

Considérons maintenant les verbes aimer et détester qui ne sont jamais perçus comme synonymes dans leurs divers emplois. Sur le plan syntaxique, leur distribution est similaire ; ils admettent tous les deux aussi bien des compléments nominaux que des compléments phrastiques :

(10) a.je déteste / aime les voyages

b.je déteste / aime voir Marie en colère

c. je déteste / aime être seul / lire

d.je déteste / aime que tu sois sportif

e. je déteste / aime quand tu mets ce parfum

Sur le plan lexical, ils admettent tous les deux des compléments renvoyant à des animés humains, des entités inertes, des événements, etc. :

(11) a. je déteste / aime ton frère

b.je déteste / aime le chocolat

c. je déteste / aime les voyages

(20) $C f$. par exemple Milner (1989: 443-446). 
Bien sûr on peut relever également des différences distributionnelles, comme dans :

(12) a. j'aimerais mieux mourir que de le rencontrer

b. ${ }^{*}$ je détesterais mieux mourir que de le rencontrer

ou bien

(13) a. j'aimerais bien un café

b. ?? je détesterais bien un café

Mais, sur les plans syntaxique et lexical, ces deux verbes ont une distribution extrêmement proche, en tout cas beaucoup plus proche que celle que l'on observe entre savoir et connaître. Des observations comparables peuvent être faites pour acheter / vendre, distributionnellement assez proches et garder/surveiller distributionnellement très différents. Nous sommes amenés à conclure qu'il n'y a pas de corrélation entre le degré de proximité distributionnelle entre deux unités et le degré de proximité entre les acceptions auxquelles donnent lieu leurs divers emplois ${ }^{(21)}$.

Enfin, la définition de la synonymie à partir du test de substitution amène à ne prendre en compte que les cas de synonymie entre des unités en relation paradigmatique (puisqu'elles doivent pouvoir commuter dans un même co-texte). Cela met à l'écart tout un pan des phénomènes de synonymie, à ma connaissance très peu étudiés, que l'on peut appeler synonymie syntagmatique, c'est-à-dire les relations de synonymie entre éléments se combinant sur 1'axe syntagmatique et ne commutant jamais sur l'axe paradigmatique ${ }^{(22)}$.

Prenons un exemple dans le domaine de la morphologie dérivationnelle, avec le préfixe RE (refaire, redire, etc.) ${ }^{(23)}$. Un certain nombre de verbes préfixés en RE peuvent, dans certains cotextes, participer à la construction d'acceptions similaires à celles obtenues avec ces verbes sans le préfixe RE :

[un chercheur faisant un exposé]

(14) a. je ferme la parenthèse et je passe au point deux

b. je referme la parenthèse et je passe au point deux

D'un point de vue distributionnel, la singularité de cette situation réside dans le fait que le préfixe RE ne commute pas avec un autre préfixe sur l'axe paradigmatique ; il commute avec rien. Il apparaît donc que nous avons affaire à un ajout : lorsqu'on ajoute le préfixe RE, en passant de (14a) à (14b), le sens de l'énoncé ne

(21) C. Kerbrat-Orecchioni (1979) avait déjà formulé des critiques similaires contre 1'hypothèse de cette corrélation ; mais, l'auteur n'abordant pas l'étude de la polysémie à ce propos, avait conclu à la dissociation entre sens d'une unité et distribution de cette unité. Il manquait à son analyse la distinction entre le plan des acceptions construites en co-texte et le plan des invariants attachés aux unités ( $c f$. ici même, le début de la section 3.1. où nous défendons l'hypothèse d'une relation, certes indirecte, entre la distribution d'une unité et sa F.S.).

(22) Je ne parle donc pas ici de ce que Auroux (1984: 95) a appelé la synonymie d'interprétation, c'est-à-dire la situation où deux unités habituellement en relation paradigmatique sont ponctuellement coordonnées sur l'axe syntagmatique dans un énoncé, comme par exemple la combinaison d'adjectifs perçus comme synonymes (... adversante et répugnante; exemple de Sénèque cité par Auroux). Le cas dont je parle ici n'est pas envisagé par Auroux.

(23) $C f$. (Jalenques, 2001). 
change pas, à quelques nuances près comme toujours. Pour cette raison, les analyses atomistes du préfixe RE considèrent que dans ce type d'emploi, le préfixe est vide de sens.

Le problème est le suivant : les cas où l'ajout du préfixe RE ne change guère le sens de l'énoncé n'apparaissent pas au hasard. Ils correspondent aux cas où l'acception construite dans l'énoncé est équivalente, à quelques nuances près, à l'une des acceptions intuitivement associées au préfixe RE :

- itération : itérer / réitérer, dupliquer/rédupliquer, doubler / redoubler (une classe), 'nouveler / renouveler (si on accepte les bases non autonomes)

- retour à une situation de référence : fermer / refermer la porte derrière soi

Considérons par exemple le verbe réitérer. Admettons que itérer renvoie à peu près à l'idée de " " faire une fois de plus qqch. »; admettons que le préfixe RE renvoie, ici, à peu près à l'idée de « modifier une situation en accomplissant une action supplémentaire ». Dans une perspective atomiste, on s'attendrait à ce que les sens de ces deux éléments s'additionnent : faire une fois de plus qqch. + faire une fois de plus qqch. = faire deux fois de plus qqch. Or, ce n'est pas ce que l'on observe :

(15) Je réitère ma demande : pourriez-vous ouvrir la fenêtre ?!

La séquence n'implique en aucun cas que l'on ait déjà répété sa demande une première fois ; elle signifie plutôt que l'on formule sa demande une seconde fois. On pourrait formuler les mêmes observations pour un verbe comme renouveler. Parce que les acceptions associées au préfixe et à la base verbale ne sont pas perçues comme « additionnées », ces verbes sont généralement considérés comme des emplois idiomatiques du préfixe (sous-entendu non compositionnel). Dans notre perspective constructiviste, ces emplois s'expliquent naturellement : la F.S. du préfixe et la F.S. de la base verbale participent, en partie de la même façon, à l'émergence d'une acception globale associée au verbe dans son ensemble. Mais ici, il n'y a pas dans le résultat interprétatif deux parties de sens perçues mais une seule ! C'est en ce sens que nous parlons de synonymie syntagmatique. Prenons une image : c'est un peu comme si vous rajoutiez une peinture d'un certain bleu dans un pot qui contient déjà de la peinture bleue ; vous ne voyez pas beaucoup changer la couleur, à quelques nuances près (l'ajout de RE change peu le sens), mais ça n'implique pas que ce que vous avez versé dans le pot soit transparent! (RE n'est pas vide de sens).

Admettons que cette analyse soit erronée et que le sens de réitérer ou renouveler ne soit pas compositionnel. Il reste alors à expliquer pourquoi cette non compositionnalité s'observe systématiquement lorsque la base verbale participe à la construction d'acceptions similaires à celles construites à partir du préfixe RE. Cette observation peut être étendue à d'autres préfixes. Par exemple, avec DÉ-, on observe une telle « idiomaticité » lorsque la base verbale est intuitivement associée à l'idée d'un éloignement, d'une séparation, de la sortie d'un espace, etc., c'est-à-dire précisément les acceptions qui peuvent être construites à partir du préfixe DÉ- (couper / découper le poulet; on vient de passer / dépasser Clermont; posez / déposez votre arme sur la table; Marc tourna / détourna le regard, etc.).

L'approche constructiviste permet ainsi de prendre en compte un autre type de compositionnalité du sens, où les contributions des unités au sens de l'énoncé ne 
correspondent pas à des parties de sens qui s'additionnent mais correspondent à la co-construction d'une même partie de sens, comme dans l'exemple (15).

Nous faisons 1'hypothèse que ce type de relations de synonymie syntagmatique que nous avons étudié à l'intérieur d'un mot (entre un préfixe et sa base) se manifeste également entre des mots. Ainsi, cette approche ouvre des perspectives sur l'analyse des paraphrases qui dans bien des cas se distinguent par l'ajout d'un ou plusieurs éléments, lesquels seraient, dans notre hypothèse, en relation de synonymie syntagmatique avec d'autres éléments de l'énoncé, comme dans :

(16) a. Il laisse une veuve et trois enfants

b. Il laisse derrière lui une veuve et trois enfants

L'ajout de l'élément derrière lui en (16b) ne change guère le sens de l'énoncé par rapport à (16a) de même que l'ajout du préfixe RE en (14b) modifie peu le sens de l'énoncé comparé à (14a). Les éléments derrière lui et laisser en (16b) participent à la co-construction d'une acception proche de celle que l'on a en (16a) sans la présence de derrière lui. Par différence avec la situation en (16), la relation entre le verbe poser et derrière lui ne met pas en jeu une synonymie syntagmatique, d'où la différence de sens entre (17a) et (17b) :

(17) a. Il a posé son chapeau

b. Il a posé derrière lui son chapeau

Il y a là, me semble-t-il, un programme de recherche à développer pour l'étude de la paraphrase, dans un cadre constructiviste.

\section{Analyse des synonymies locales entre les verbes garder et laisser, dans le cadre d'une sémantique constructiviste}

\subsection{Présentation des verbes garder et laisser}

Nous ne proposerons pas une analyse sémantique approfondie de ces deux verbes. Notre objectif est avant tout de fournir les éléments d'information minimaux pour comprendre le fonctionnement sémantique général de ces verbes, l'enjeu étant d'utiliser les hypothèses sur ces verbes pour proposer une analyse constructiviste de l'émergence locale d'une synonymie entre deux énoncés contenant ces verbes. Bref, les justifications pour l'analyse proposée de ces verbes seront minimales, mais elles nous paraissent importantes pour éviter que notre analyse de la synonymie entre deux énoncés contenant garder/laisser ne paraisse ad hoc.

D'autre part, avant de présenter ces invariants, je voudrais préciser la façon dont ils sont obtenus. Nous avons vu dans la section précédente que la distribution d'une unité n'est pas corrélée à telle ou telle de ses acceptions. D'autre part, il n'y a pas deux unités dans une langue qui aient exactement la même distribution ( $c f$. les travaux du LADL). Une distribution (entendue au sens de l'ensemble des cotextes d'emplois possibles pour cette unité) est donc spécifique à l'unité. Elle est liée à ce qui constitue l'identité de l'unité elle-même. En conséquence, si l'on doit envisager un lien impliquant la distribution, ce n'est pas avec telle ou telle acception, mais c'est avec la forme schématique elle-même. C'est précisément sur cette hypothèse que reposent les analyses de forme schématique dans la T.O.P.E., du moins dans l'orientation que je défends. Dans son principe, la mise au jour d'une 
F.S. passe par l'observation non pas des acceptions construites à partir des emplois de l'unité considérée mais passe par l'observation des contraintes sur la construction de ces acceptions, ce qui implique l'observation des contraintes sur la distribution du mot ${ }^{(24)}$. C'est à partir de ce type de contraintes que nous justifierons minimalement notre analyse de garder et laisser.

C'est parce que la F.S. est élaborée à partir de l'observation de ces contraintes que, contrairement à ce qu' affirme Kleiber (1997 : 42), par principe une F.S. n'est pas trop puissante (au sens où elle prédirait la possibilité d'acceptions non attestées pour le mot considéré). Kleiber (2008 : 92), évoquant un travail de Martin $(2005)^{(25)}$ donne l'exemple des différentes acceptions du mot quartier (quartier d'orange, de viande, de lune, quartier d'une ville, le quartier des femmes, etc.) dont on peut certes "subsumer » intuitivement une propriété générale "partie d'un tout », mais celle-ci est trop puissante ; elle prédit la possibilité d'emplois non attestables désignant pourtant la partie d'un tout: quartier d'un livre (pour le chapitre d'un livre) ou bien quartier d'une symphonie pour désigner le mouvement d'une symphonie. Selon nous, cette hypothèse sur quartier est trop puissante en raison même de la façon dont elle a été élaborée : c'est parce qu'elle a été construite de façon intuitive à partir de l'observation des acceptions attestées. Dans notre démarche, l'analyse pour élaborer une F.S. de quartier s'appuierait au contraire sur l'observation du caractère non attestable de quartier de livre / d'une symphonie, mais aussi quartier d'un meuble, d'une maison, d'une voiture etc. C'est l'observation et l'analyse des contraintes d'emploi elles-mêmes qui permettent d'élaborer un invariant qui ne soit pas trop puissant. On ne trouve pas l'identité du mot dans un hypothétique noyau central sous-déterminé mais on la trouve au contraire à la périphérie des emplois du mot.

\subsubsection{Présentation de quelques emplois du verbe garder}

En simplifiant, on peut ranger les emplois du verbe garder en deux grandes classes, illustrées par les quelques exemples suivants :

(18) a. Paul a gardé ses chaussures pour traverser la rivière

b. on préfère garder les volets fermés à cause de la chaleur

c. le capitaine a décidé de garder Marie dans son équipe

(19) a. hier soir, François a gardé les enfants de la voisine

b. le pont est bien gardé

c. enfant, Eric gardait les moutons avec son grand-père, pendant l'été

\section{Convention de notation:}

on notera par les lettres majuscules $\mathrm{X}$ et $\mathrm{Y}$ respectivement les premier et deuxième arguments du verbe :

$$
\frac{\text { Paul a gardé son manteau }}{\mathrm{X}}
$$

Dans la première classe d'emplois, garder donne lieu à des interprétations don-

(24) Pour une présentation développée de la démarche empirique permettant la mise au jour d'une F..S., je renvoie à Jalenques (à par. 2010).

(25) Martin, Robert (2005) : «Traitement automatique de la polysémie. Eloge du dictionnaire», La polysémie, Soutet, O. (éd.), Paris : Presses de l’Université Paris-Sorbonne, pp. 167-173. 
nées comme proches de conserver ou maintenir. Dans la deuxième classe, l'emploi du verbe est proche de s'occuper de, surveiller.

Nous défendons l'idée que le verbe garder met en jeu dans tous ces emplois un même scénario abstrait fondé sur une relation de localisation. Nous entendons par localisation une relation posant que 1'existence d'une entité B est rapportée à l'existence d'une entité A. Par existence, nous entendons le fait qu'une entité est envisagée comme étant le cas (à un moment donné dans une situation donnée). C'est par exemple la différence entre Paul a un manteau et Paul aimerait avoir un manteau. La relation exprimée par garder est toujours une relation effective, actualisée et non une relation envisagée.

Selon les co-textes, cette relation de localisation participe à la construction de relations très variables, la localisation spatiale n'en étant qu'une interprétation possible (Vous êtes priez de garder votre titre de transport sur vous) ; elle peut aussi participer à la construction contextuelle d'une relation d'appartenance (ils ont gardé un petit pied-à-terre à Paris).

Nous pouvons maintenant présenter notre hypothèse de F.S. pour le verbe garder:

Le verbe garder exprime une relation de localisation d'un terme B relativement à un terme A de telle sorte que :

- cette localisation constitue une identité circonstancielle de B,

- cette localisation se manifeste relativement à une situation où l'on s'attend à ce que cette relation $\mathrm{A}-\mathrm{B}$ puisse cesser,

- par rapport à ce changement envisagé, cette relation A-B perdure dans cette situation, du fait de A.

Dans le cas le plus simple, les termes A et B correspondent respectivement aux arguments $\mathrm{X}$ et $\mathrm{Y}$ de la relation prédicative, comme dans 1'exemple Paul a gardé ses chaussures pour traverser la rivière, où $\mathrm{A}=\mathrm{X}=$ Paul et $\mathrm{B}=\mathrm{Y}=$ ses chaussures. Pour mettre en lumière la F.S. de garder, comparons les deux séquences suivantes :

(20) a. n'aie pas peur, Paul te tient

b. n'aie pas peur, Paul te garde

On est dans une situation où l'on s'attend à ce que la relation entre X et Y (Paul et l'interlocuteur) puisse cesser; en même temps, on comprend que la relation perdure du fait de Paul. Il y a cependant une différence cruciale entre les deux séquences. En (20a), la relation s'interprète typiquement comme un lien physique : par exemple, dans une randonnée, on traverse une rivière en marchant sur un tronc couché, on s'adresse à un enfant qui a peur de tomber en lui disant « $n$ 'aie pas peur, Paul te tient ». Ce type d'interprétation est bloquée avec garder. En (20b), ce verbe impose un contexte du type Paul te garde comme membre de son équipe, Paul te garde dans son entreprise en tant qu'employé de cette entreprise, etc. Bref, Y est gardé par Paul en tant qu'employé, en tant que membre de l'équipe, etc. C'est cela que nous appelons une "identité circonstancielle » de Y. Cette identité est « circonstancielle » parce qu'elle résulte des circonstances de la relation de Y à X. Je veux dire que cette identité est construite à partir de la relation de $\mathrm{Y}$ à $\mathrm{X}$.

C'est cette dimension de la F.S. de garder qui participe à la construction de la valeur « sur soi » que nous avions relevée pour (5a) dans garder son manteau à la cérémonie. L'identité circonstancielle de manteau, liée donc à X, est ici du type 
« sert à protéger $\mathrm{X}$ du froid». De même qu'en (20b) Paul est en relation avec X en tant qu'employé, en (5a), manteau est en relation avec $\mathrm{X}$ en tant qu'il protège $\mathrm{X}$ contre le froid. La valeur «sur soi » émerge comme conséquence de cette identité circonstancielle de manteau ; c'est en ce sens qu' elle est construite. Pour revenir à (20b), ce mode de construction de l'identité circonstancielle distingue garder d'un verbe comme conserver, qui lui aussi met en jeu une identité circonstancielle de $\mathrm{Y}$, mais celle-ci n'est pas constituée à travers la relation de $\mathrm{Y}$ à X; d'où l'aspect moins naturel d'une séquence comme? n'aie pas peur, je te conserve. On voit ici dans quelle mesure la F.S. de garder ne se réduit pas à un simple « chapeau sémantique » du type « continuer à avoir».

Cette mise en jeu d'une identité circonstancielle de Y se retrouve de façon explicite dans 1'affinité de garder avec la construction à attribut de l'objet : garder les volets fermés / garder son prestige intact / garder qqn prisonnier / garder les genoux fléchis / les yeux baissés / le bras tendu, etc. De plus, dans une situation où la relation entre $\mathrm{X}$ et $\mathrm{Y}$ est purement spatio-temporelle, ne mettant en jeu aucune identité circonstancielle de Y liée à X, l'emploi de garder n'est pas possible :

[au pied d'un escalier roulant]

(21) a. prière de tenir la main courante

b. ?? prière de garder la main courante

Je terminerai la présentation de garder en mettant en lumière le scénario abstrait pour la deuxième classe d'emplois, à partir de la comparaison des séquences suivantes :

(22) a. Alain est gardé par deux policiers

b. Alain est surveillé par deux policiers

La séquence (22b) n'implique nullement que les déplacements de Alain soient limités par les policiers. Il peut s'agir d'une filature par exemple. On pourra dire que Alain est surveillé à son insu. Les policiers n'empêchent pas que Alain aille où bon lui semble. Au contraire, la séquence (22a) implique une situation où Alain n'est pas libre de ses mouvements. Il est contraint de rester à proximité des policiers, il ne peut pas s'en éloigner. Nous retrouvons donc une relation de localisation, ici construite dans le domaine spatial, entre $\mathrm{X}$ et $\mathrm{Y}$. Il suffit de produire un co-texte induisant que Y est libre de ses mouvements pour que l'emploi de garder soit bloqué :

(23) a. Deux inspecteurs surveillaient les allers et venues du suspect

b. * Deux inspecteurs gardaient les allers et venues du suspect

De plus, la séquence (22a) ci avant induit une situation où Y pourrait s'échapper, c'est-à-dire une situation où Alain n'est pas enfermé dans une cellule. Nous sommes donc dans une situation où l'on envisage a priori que la relation de localisation X-Y puisse cesser, du fait de Y. De même, on parle de garder des enfants lorsque les parents sont absents ; on parle de garder les moutons quand il n'y a pas de barbelés. La relation X-Y joue simultanément sur deux plans : d'une part faire en sorte que Y ne s'éloigne pas; d'autre part, faire en sorte que l'état de Y dans le cadre de sa relation à $X$ ne change pas (que Alain reste prisonnier; que l'enfant ne se fasse pas mal, qu'il ne lui arrive rien; que le mouton ne se blesse pas, ne rencontre pas un prédateur, etc.). On retrouve donc la mise en jeu d'une identité circonstancielle de Y. On peut vérifier cette dimension de la F.S. de garder en comparant les deux séquences suivantes : 
(24) a. Alain est gardé par deux policiers

b. Alain est tenu par deux policiers

La séquence (24b) n'implique aucune identité particulière de $\mathrm{Y}$ dans sa relation à X ; Alain peut par exemple être un accidenté de la route soutenu par les deux policiers. Avec garder, la relation de Alain à policier induit nécessairement une certaine image de Alain, la plus naturelle avec policier étant qu'il est prisonnier.

Bien sûr, au gré des propriétés notionnelles de $\mathrm{X}$ et de $\mathrm{Y}$, cette identité circonstancielle de Y sera extrêmement variable. De ce point de vue, ce que fait garder dans l'énoncé, c'est d'imposer la construction de la représentation d'une identité circonstancielle de Y, quelle qu'elle soit.

\subsubsection{Présentation de quelques emplois du verbe laisser}

A l'étape actuelle de mes recherches, je n'ai pas de caractérisation invariante aboutie à proposer pour ce verbe. Néanmoins, la description non aboutie que je développe ci-après sera suffisante pour permettre l'analyse constructiviste de la synonymie locale entre les acceptions émergeant des emplois de garder et laisser. Partons de l'exemple suivant:

(25) les manifestants ont laissé passer la délégation

Comparons-le avec la séquence négative suivante :

(26) les manifestants n’ont pas laissé passer la délégation

Cette séquence signifie que le procès délégation-passern'a pas lieu. Cela nous conduit à poser pour (25) qu'à travers le procès construit en lien avec laisser l'existence du procès délégation-passer est en jeu. Premier point donc, laisser marque la construction de l'existence d'un terme; notons-le T. Ici, T correspond à délégation-passer. Précisons que la prédication d'existence d'un terme suppose toujours une situation par rapport à laquelle est prédiquée cette existence. Notonsla S. Laisser marque donc la construction de l'existence d'un terme T dans une situation S. Le type de terme dont l'existence est en jeu est très variable :

(27) a. la prédication d'existence d'un état:Paul a fini par laisser sa sœur tranquille

b. la prédication d'existence d'une relation : Paul a laissé les clés au concierge

c. la prédication d'existence de Y lui-même : les voleurs ont laissé des traces

A chaque fois, on constate que la négation du procès laisser entraîne la négation de l'existence du terme en jeu :

(28) a. Paul n'a pas laissé sa sœur tranquille

b. Paul n'a pas laissé les clés au concierge

c. les voleurs n'ont pas laissé de traces

En (28a), on comprend que dans la situation considérée, la sœur n'est pas tranquille ; en (28b), on comprend que le concierge n'a pas les clés ; en (28c), on comprend qu'il n'y a pas de traces.

De plus, dans chaque cas, l'existence du terme T dépend de X (le premier argument du verbe). La spécificité et la complexité du fonctionnement sémantique de laisser résident dans la façon dont se constitue cette dépendance. Reprenons l'exemple (25) et comparons l'interprétation à celle obtenue avec le verbe regarder : 
(25) a. les manifestants ont regardé passer la délégation

b. les manifestants ont laissé passer la délégation

Le contraste interprétatif montre qu'avec laisser on part d'une situation où les manifestants font a priori obstacle au passage de la délégation. Plus précisément, c'est la présence des manifestants dans la situation $\mathrm{S}$ qui fait a priori obstacle au passage de la délégation : par exemple, les manifestants occupent toute la largeur de la rue. Du coup, on interprète la séquence comme « les manifestants n'ont pas fait obstacle au passage de la délégation ». Donc, a priori, les manifestants pourraient empêcher le passage de la délégation et ils ne le font pas. Désignons par P ce procès envisagé. Dans la situation $S$, le procès $P$ est absent (puisqu'il n'a pas lieu). Le point essentiel est que $X$ n'est présent relativement à $T$ que du point de vue d'une absence ${ }^{(26)}$. Il est intéressant ici d'observer que la plupart des gloses synonymiques fournies par les dictionnaires pour les différentes acceptions construites dans les énoncés contenant laisser comportent une négation; en voici quelques exemples tirés du Petit Robert (2007) ; les exemples ne sont pas tous ceux du dictionnaire :

(29) a. ne pas empêcher: les manifestants ont laissé passer l'ambulance

b. ne pas intervenir: je vous laisse choisir

c. ne plus importuner : Paul a enfin laissé sa sæur tranquille

d. ne pas prendre : il a laissé toute la croûte

e. ne pas emmener avec soi : il a laissé son manteau au vestiaire

f. ne pas faire soi-même : il me laisse toutes les corvées

g. ne pas priver de : laissons-lui une chance

h. ne pas supprimer : il a laissé des fautes dans son texte

Cela indique que, pour ces emplois, $\mathrm{X}$ est toujours présenté comme n'étant pas la source ${ }^{(27)}$ d'un procès $P$. L'actualisation de ce procès aurait pour effet la non existence de T. Si X (les manifestants) actualise le procès empêcher le passage, alors le procès délégation-passer n'est pas actualisé. Il y a donc trois dimensions qui jouent simultanément :

i) on s'attend a priori à ce que ce procès soit actualisé (par exemple les manifestants sont hostiles à la délégation)

ii) le procès $P$ n'est pas actualisé

iii) la non actualisation de $\mathrm{P}$ a pour effet $\mathrm{l}$ 'actualisation de $\mathrm{T}$ en $\mathrm{S}$

d'où, dans cette classe d'emplois, l'intuition que les manifestants concèdent le passage. On emploierait difficilement laisser pour référer à un contexte où les manifestants ne font pas a priori obstacle au passage de la délégation.

En (29d), on s'attend a priori à ce que X mange la croûte, par exemple la croûte d'une tarte (procès $\mathrm{P}$ ) et en même temps on constate que P n'est pas actualisé. C'est le fait que $X$ n'actualise pas le procès $P$ qui a pour effet (le maintien de) l'existence de la croûte dans la situation S considérée (par exemple dans l'assiette).

(26) Sans entrer dans le débat ici, notons que cette analyse est assez différente de celles généralement proposées dans les analyses grammaticales de la causalité qui présentent le verbe « laisser » comme un marqueur de causalité faible ( $c f$. par exemple Mœschler, 2003).

(27) Je parle de source du procès plutôt que d'agent car les procès en question sont très variables et n'impliquent pas toujours que le premier argument corresponde à un agent. 
En fait, il y a donc deux cas : soit le procès $\mathrm{P}$ est simplement envisagé relativement à $X$ et n'est pas actualisé, comme dans (25) (on envisage un procès qui n'a pas lieu, relativement à $\mathrm{X}$ ), soit on part d'une situation où le procès $\mathrm{P}$ est actualisé et la construction de la non actualisation de ce procès s'interprète alors comme la cessation de ce procès (on passe de $\mathrm{P}$ à non $\mathrm{P}$ ), comme en $(29 \mathrm{~d})$. De ce point de vue, la glose du dictionnaire pour (29d) est en partie inexacte : ce n'est pas « ne pas prendre », c'est « ne plus prendre (manger) de »; d'où l'intuition que la croûte dans l'assiette correspond à ce qui résulte de la cessation du procès manger ; alors qu'en (25), le passage de la délégation correspond à ce qui résulte de la non actualisation du procès faire obstacle. Considérons maintenant (29c) qui relève du même cas que (29d) :

(29) c. Paul a enfin laissé sa sœur tranquille

L'interprétation de cette séquence est proche de Paul a cessé d'embêter sa sœur. On part d'une situation où $\mathrm{X}$ est source du procès $\mathrm{P}$ embêter-sœur actualisé qui a pour résultat que la sœur n'est pas tranquille. Le fait que le procès $X$-embêtersœur cesse a pour effet l'actualisation de T (sœur-être tranquille). Là encore, l'existence de $\mathrm{T}$ ( sœur-être tranquille) découle de la présence de $\mathrm{X}$ relativement au fait que $\mathrm{X}$ n'actualise pas le procès embêter sa sæur dont il constitue la source potentielle. Etant donné que l'on part d'une situation où $\mathrm{P}$ est déjà actualisé, la glose de l'énoncé ne correspond pas à «ne pas importuner la sœur », mais correspond à « ne plus importuner la sœur ». Cette hypothèse d'après laquelle X n'est présent relativement à $\mathrm{T}$ que du point de vue de son absence d'un procès repose notamment sur l'observation que les compléments qualifiants possibles sont ici toujours associés à une non action sur $\mathrm{Y}$ de la part de $\mathrm{X}$, comme dans laissez-moi seule, laissez-moi en paix, etc. On aura plus difficilement :

(30) ?? Paul l'a laissée embêtée pendant cinq minutes

Considérons maintenant l'exemple suivant :

(31) les voleurs ont laissé des traces

Dans la même série que (31), nous avons des emplois du type laisser des indices, laisser une empreinte, laisser une marque, etc. Ici, le terme T correspond à Y luimême (les traces). L'interprétation de la séquence implique a priori que les voleurs ne sont plus là. X n'est plus présent dans la situation S. La manifestation de la présence de $\mathrm{X}$ relativement à $\mathrm{T}$ se ramène à une absence. Remarquons que dans cette dernière classe d'emplois, les noms en position de complément de laisser mettent en jeu l'idée d'une absence. En effet, qu'est-ce qu'une trace si ce n'est la manifestation de la présence d'une entité qui n'est pas là (on peut penser à la théorie des traces en grammaire générative). La particularité de cette classe d'emplois est liée à la spécificité du procès $\mathrm{P}$ associé à $\mathrm{X}$ : il correspond à $\mathrm{X}$-être présent. D'ailleurs, une paraphrase très proche de (31) serait :

(32) les voleurs ont laissé des traces de leur passage

Le terme de source (du procès $\mathrm{P}$ ) est trop spécifique aux emplois vus jusqu'à maintenant, car dans d'autres cas, $\mathrm{X}$ lui-même correspond à un procès. Dans ce cas, le procès $\mathrm{P}$ est identifié à $\mathrm{X}$ :

(33) a. le cyclone Dean a laissé des traces 
b. le coup a laissé des marques

c. sa remarque me laisse une drôle d'impression

Le point crucial est que du point de vue de la construction de la représentation aspectuelle du procès $\mathrm{P}$, celui-ci est nécessairement terminé, dans sa relation à $\mathrm{T}$ (les traces, les marques). On retrouve donc le même mécanisme interprétatif que précédemment : on se trouve dans une situation $S$ après le passage du cyclone; $\mathrm{P}$ n'est plus actualisé, donc le procès désigné par X est absent de la situation $\mathrm{S}$. D'ailleurs, 1'emploi du présent est moins naturel ici : ? le cyclone Dean est en train de laisser des traces. Encore une fois, T correspond à ce qui résulte de la cessation du procès $P$.

En conséquence, selon l'interprétation de $\mathrm{P}$ ou bien selon l'interprétation de $\mathrm{X}$ (il peut correspondre à un procès), l'absence du procès $\mathrm{P}$ dans la situation $\mathrm{S}$ peut avoir pour effet une absence liée à $\mathrm{X}$ lui-même dans la situation $\mathrm{S}$, comme dans l'exemple suivant :

(34) sa mort laisse un vide terrible

On aura plus difficilement :

(35) ? sa naissance laisse un bonheur formidable

car on a plus de mal à construire la représentation d'un contexte où une naissance mettrait en jeu une absence ! Revenons à (34). Là encore, l'énoncé renvoie à une situation où a priori on envisage que la personne ne meurt pas (procès $\mathrm{P}=X$-être en vie), soit parce que cette mort n'est pas souhaitée soit parce qu'on ne s'y attend pas. Cette analyse repose sur l'observation qu'une séquence comme il laisse une veuve et trois enfants évoquera plus naturellement un contexte où la personne n'était pas en âge de mourir plutôt qu'une situation où il s'agit d'une personne très âgée.

En résumé, par hypothèse, le verbe laisser mmarque la construction de :

- l'existence d'un terme T

(délégation-passer / sœur-être tranquille / des traces)

- dans une situation $\mathrm{S}$

(la rue / la chambre de la sœur / le lieu du cambriolage)

- relativement à un terme $\mathrm{X}$

(les manifestants / le frère / les voleurs / le cyclone)

- $\mathrm{X}$ est associé à un procès $\mathrm{P}$

(empêcher / importuner / souffler en tempête)

- P n'est pas actualisé dans la situation $\mathrm{S}$

(les manifestants ne font pas obstacle au passage /

le frère n'embête plus sa sœur /

le cyclone est passé)

\subsection{Analyse de l'émergence d'une synonymie locale entre deux énoncés contenant garder ou laisser}

A l'issue de la brève présentation de ces deux verbes, il apparaît qu'ils ont un fonctionnement sémantique nettement différent. Dans un cadre constructiviste, la question de la synonymie prend alors la forme suivante : comment se fait-il que 
des unités dont les formes schématiques sont aussi différentes puissent participer à la construction en co-textes d'acceptions perçues comme synonymes ${ }^{(28)}$ ? Pour répondre à cette question nous allons examiner les facteurs qui contribuent à l'émergence de cette synonymie locale à un de leurs emplois. Reprenons les deux séquences suivantes, perçues comme synonymes, évoquées au début de l'article :

(1) a. On préfère garder les volets fermés à cause de la chaleur

b. On préfère laisser les volets fermés à cause de la chaleur

La première caractéristique la plus évidente de cet emploi est d'ordre syntaxique : nous avons affaire à un attribut du COD, ainsi que le confirme le test de la pronominalisation :

(36) a. On préfère les garder fermés à cause de la chaleur

b. On préfère les laisser fermés à cause de la chaleur

Notons les différents syntagmes de la structure syntaxique par les lettres X, Y et Z:

(1a-b) On préfère garder / laisser les volets fermés à cause de la chaleur

Le premier aspect qui favorise l'émergence d'une proximité interprétative concerne la relation Y-Z. Du point de vue de la F.S. de garder, l'élément Z constitue l'identité circonstancielle actualisée dans le cadre de la relation X-Y. Du point de vue de laisser, la relation Y-Z (volets-être fermés) constitue le terme T dont l'existence dépend de X. Ainsi, le premier élément qui favorise l'émergence d'une synonymie réside dans le fait que le terme $\mathrm{T}$ dont l'existence est en jeu avec laisser correspond à une identité circonstancielle, ce que n'impose en aucune façon ce verbe (cf. laisser passer, laisser des traces, etc.), mais qui correspond à ce que garder impose.

Le deuxième facteur favorisant la construction d'une proximité interprétative entre (1a) et (1b) découle de l'interprétation de la relation entre X et Y. Dans la F.S. de garder, Y est localisé par rapport à X. Nous avions vu que cette localisation pouvait participer à la construction de relation très diverses (avoir sur soi : garder ses papiers d'identité ; être possesseur de : garder un pied-à-terre à Paris, etc.). Cette relation de localisation est plus ou moins saillante selon les emplois. Dans le cas présent, elle est peu saillante et se réduit à : la situation définie par « volets-fermés » est rapportée à X en tant qu'il est l'usager / le bénéficiaire de cette situation ; par exemple, il fait plus frais à l'intérieur de la maison.

Cette saillance faible de la relation de localisation entre $\mathrm{X}$ et $\mathrm{Y}$ du point de vue de garder est indispensable à l'émergence d'une synonymie avec l'emploi de laisser puisque ce dernier met en jeu au contraire une absence de $\mathrm{X}$ dans sa relation à Y. Du point de vue de laisser, l'absence de X n'est pas saillante dans la mesure où elle se ramène à une absence d'agentivité relativement au procès $\mathrm{P}$ ouvrir-volets ; cette absence de X par rapport à T n'implique pas une absence de X lui-même dans la situation S (absence que 1'on avait par contre dans les voleurs ont laissé des indices qui suppose que les voleurs sont partis).

(28) Le fait que je m'attache à décrire dans cet exemple la construction de la proximité sémantique entre les deux énoncés ne doit pas occulter le fait qu'il s'agit, comme toujours, d'une synonymie partielle. Pour une analyse dans le même cadre théorique, s'attachant au contraire à analyser les différences sémantiques entre des emplois synonymes, je renvoie à Thuillier (2004). 
C'est donc la conjonction de deux facteurs différents concernant la relation XY qui contribue à l'émergence de la synonymie :

- pour garder, la non saillance de la relation de localisation de Y à X ;

- pour laisser, le fait que l'absence de X par rapport au procès $\mathrm{P}$ ouvrir-volets n'implique pas 1'absence de X lui-même dans la situation S.

Du point de vue de garder, il suffirait de choisir comme second argument du verbe un terme qui renvoie explicitement à sa relation de localisation à $X$, rendant saillante cette localisation, pour que la substituabilité entre les deux verbes devienne moins naturelle, par exemple si on prend un nom de partie du corps :

(37) a. Paul avait du mal à garder les yeux ouverts

b. ?? Paul avait du mal à laisser les yeux ouverts

Le troisième facteur qui favorise la proximité sémantique en marginalisant la relation de $\mathrm{Y}$ à $\mathrm{X}$ découle du fait que la relation $\mathrm{Y}-\mathrm{Z}$ est préconstruite, ainsi que le montre l'emploi de la négation :

(38) a. Ils n'ont pas laissé les volets fermés

b. Ils n'ont pas gardé les volets fermés

Avec les deux séquences, on comprend qu'ils ont ouverts les volets, ce qui implique qu'au départ, dans la situation $\mathrm{S}$, les volets sont fermés. Le fait que la relation $\mathrm{Y}-\mathrm{Z}$ soit préconstruite réduit $\mathrm{X}$ à un rôle de valideur d'un état de chose déjà établi. C'est toute la différence avec laisse ta sœur tranquille où au contraire, la tranquillité de la sœur n'est justement pas établie. Là encore, la substitution avec garder n'est pas possible (?? garde ta sæur tranquille).

Le quatrième et dernier facteur favorisant la construction de la proximité interprétative découle de la présence du verbe préférer et du complément à cause de la chaleur. Dans 1'emploi garder / laisser les volets-fermés, avec garder, X est l'agent d'un non changement ( $\mathrm{X}$ veut et fait en sorte que les volets ne soient pas ouverts) alors qu'avec laisser, $\mathrm{X}$ est le non agent d'un changement (X n'accomplit pas l'action qui aboutirait à volets-ouverts). Cela revient au même du point de vue de l'état des volets, mais cela ne revient pas au même du point de vue de l'interprétation de $\mathrm{X}$ : il a une dimension agentive avec garder mais pas avec laisser (je simplifie). Dès lors, laisser n'impose pas que X s'interprète comme ayant l'intention que les volets restent fermés. Il peut les avoir laissés fermés par négligence, parce qu'il a oublié de les ouvrir, etc. La présence du verbe préférer, combiné au complément à cause de la chaleur, conduit donc à construire une représentation de $\mathrm{X}$ comme voulant que les volets restent fermés, ce qui neutralise la différence de statut de $\mathrm{X}$ avec laisser par rapport à garder.

Dès lors que l'on produit un co-texte qui met en saillance la non agentivité de $\mathrm{X}$ du point de vue de laisser, alors la synonymie avec le même co-texte associé à garder est beaucoup moins nette :

(39) a. Regarde ! Ton grand-père a encore laissé le gaz allumé

b. ? Regarde! Ton grand-père a encore gardé le gaz allumé

Il apparaît donc que ce sont tous les éléments du co-texte en (1a) et (1b) qui contribuent à l'émergence d'une synonymie locale perçue entre deux « parties » du sens global de deux énoncés se distinguant par la présence de garder ou laisser. 


\section{Conclusion}

Nous voudrions clore cet article en abordant un point non discuté jusqu'ici, à savoir la nature de la différence sémantique entre les acceptions perçues comme synonymes, c'est-à-dire les « quelques nuances de sens » qui les distinguent.

Dans le co-texte on préfère garder / laisser les volets fermés à cause de la chaleur, nous avons noté que la représentation construite pour le premier argument du verbe n'était pas la même : avec garder, il correspond à l'agent d'un non changement (on ne veut pas que les volets soient ouverts), alors qu'avec laisser il correspond au non agent d'un changement (on n'est pas agent de l'ouverture des volets). Du point de vue de l'interprétation globale de l'énoncé, cette différence peut devenir secondaire dans la situation où l'enjeu communicatif du locuteur concerne l'état des volets (le fait qu'ils demeurent fermés). Mais du point de vue des verbes garder et laisser, cette différence est essentielle puisqu'elle renvoie, indirectement, à un des aspects de leur forme schématique.

Ainsi, les nuances de sens différenciant les acceptions synonymes peuvent être à la fois secondaires du point de vue du sens global des énoncés où elles se manifestent et essentielles du point de vue des mots qui participent à l'émergence de ces nuances.

\section{Références citées}

Auroux, S. (1984) : «D'Alembert et les synonymistes », Dix-huitième siècle, 16, pp. $93-108$

Culioli, A. (1990-1999) : Pour une linguistique de l'énonciation (3 vol.). Paris : Ophrys.

De VoguË, S., PAillard, D. (1997) : «Identité lexicale et hétérogénéité de la variation co-textuelle. Le cas de suivre», Table ronde, Université de Caen, 2-3 février 1996, in: Co-texte et calcul du sens (ed. C. Guimier), Presses Universitaires de Caen, pp. 41-62.

DUCHÀCEK, O. (1964) : «Contribution à l'étude de la sémantique : Les synonymes 》, Orbis XIII(1), 35-49.

FRANCKEL, J.-J. (2002) : «Introduction », Langue Française, 133, pp. 3-15. - (2005): «De l'interprétation à la glose : vers une méthodologie de la reformulation », Actes du colloque " D'une langue à l'autre », Daniel Lebaud (éd.), Besançon, 5-7 septembre 2002, Presses Universitaires de Franche-Comté, pp. 51-78.

FRANCKeL, J.-J., LeBAUd, D. (1990) : Les figures du sujet, Paris : Ophrys.

FrançOIS, J., VictorRi, B., MANGUIN, J.-L. (2003) : « Polysémie adjectivale et synonymie : l'éventail des sens de curieux », La polysémie, Soutet, O. (ed.), Paris : Presses de l'Université de la Sorbonne.

FUCHS, C. (1980) : «Synonymie de mots autrefois, synonymie de phrases aujourd'hui », Modèles linguistiques, II/2, pp. 5-21.

- (1992) : «Modulations qualitatives sur l'itération. Les emplois concurren- 
tiels de encore et re- ", dans La quantité et ses modulations qualitatives, Actes du colloque « Linguistique et Sémiotique II », Limoges, 28-30 mars 1991, J. Fontanille (éd.), Pulim / Benjamins : Limoges / Amsterdam / Philadelphia, pp. 129-142.

- (1994): Paraphrase et énonciation, Paris : Ophrys.

- (2007) : «Relations de synonymie entre polysèmes : le réseau comme-manière-façon », Le Français Moderne, LXXV :1 («La Synonymie », F. Berlan, ed.), pp. 97-113.

Gosselin, L. (1996) : «Le traitement de la polysémie contextuelle dans le calcul sémantique », Intellectica, 22, pp. 93-117.

Gross, M. (1997) : «Synonymie, morphologie dérivationnelle et transformations ", Langages, 128, pp. 72-90.

HARRIS, Z. S. (1954) : « Distributional Structure », Word, 10 :2/3, pp. 146-162.

JALENQUES, P. (2001) : «Quand la diachronie renvoie à la synchronie : Étude des emplois idiomatiques du préfixe RE en français (renier, remarquer, regarder, etc.) », Recherches linguistique de Vincennes, 30, pp. 39-61.

— (à par. 2010) : "Analyse sémantique et contraintes distributionnelles : l'exemple du verbe monter », Actes du XXV Congrès International de Linguistique et Philologie Romane, 03-08 septembre 2007, Université d'Innsbruck.

Kerbrat-OrecCHIONI, C. (1979) : De la sémantique lexicale à la sémantique de l'énonciation, thèse d'Etat, soutenue en 1977, Université Lyon II.

KleIBer, G. (1999) : Problèmes de sémantique. La polysémie en question, Villeneuve d'Ascq : Presses Universitaires du Septentrion.

— (2008) : «Petit essai pour montrer que la polysémie n'est pas un sens interdit », actes du Congrès Mondial de Linguistique Française, Paris, juillet 2008, pp. 87-101

[accessible à l'adresse suivante : http ://www.linguistiquefrancaise.org/index.php ?option $=$ article $\&$ access $=$ doi\&doi $=10.1051 / \mathrm{cmlf08341}]$

LIPSHITZ, E. (1978) : «Réflexions sur la synonymie lexicale », Cahiers de lexicologie, XXVII/1, 103--117.

MilneR, J.-C. (1989) : Introduction à une science du langage, Paris : Seuil.

Moeschler, J. (2003) : «L'expression de la causalité en français », Cahiers de Linguistique Française, 25, 11-42.

SANCHEZ, J. et CADIOT, P. (2007) : « Le cas des substantifs de couleur construits. $\mathrm{X}_{\mathrm{A}} \rightarrow{ }_{\text {suf.eur }} \mathrm{Xeur}_{\mathrm{N}} / \mathrm{X}_{\mathrm{A}} \rightarrow{ }_{\text {conv }} \mathrm{X}_{\mathrm{N}}$ : un point de vue sur la synonymie », Le français moderne, 75/1, pp. 114-140.

SCHREYER, R. (1976) : «Synonyms in Context», Trier : Linguistic Agency University of Trier. (Repr. Working Papers in Language and Linguistics 7 (1978) : 8-21). [consulté dans sa version pdf : http ://www-users.rwth-aachen.de/Ruediger.Schreyer/3 frames.htm]

THUILleR, F. (2004) : "Synonymie et différences : le cas de paraître et sembler », Le verbe dans tous ses états. Grammaire, sémantique, didactique, actes de la journée d'étude « Didactique de la syntaxe », 29 janvier 2004, Dyptique, 2, Namur : Presses Universitaires de Namur, pp. 161-178. 